\title{
Intraocular pressure outcome after phacoemulsification and intraocular lens implantation in chronic angle closure glaucoma patients without extensive peripheral anterior synechiae
}

\author{
Kanokorn Sakpisuttivanit, Visanee Tantisevi \\ Department of Ophthalmology, Faculty of Medicine, Chulalongkorn University \\ Bangkok 10330, Thailand
}

\begin{abstract}
Background:Cataract extraction surgery significantly decreases intraocular pressure (IOP) in patients with various forms of angle closure glaucoma (ACG). However, most reports did not specify the circumferential extent of peripheral anterior synechiae (PAS).

Objective: Evaluate IOP after phacoemulsification with intraocular lens (IOL) implantation in chronic ACG patients without extensive PAS.

Materials and methods: Data of chronic ACG patients with documented peripheral PAS $\leq 180$ degrees and clinically significant cataract, who underwent phacoemulsification with IOL implantation at King Chulalongkorn Momorial Hospital between May 2002 and June 2007 were reviewed retrospectively. Baseline characteristics as gender, degree of PAS, IOP, and number of medications were collected. The outcomes were post-operative IOP at 6,12 , and 18 months, and number of hypotensive medications.

Results: Twenty-five eyes from 25 patients (mean age: 73.0 years old) were included in this study. Twenty-three out of 25 patients were female, and 17 had PAS less than 90 degrees. Pre-operative mean IOP was $15.6 \mathrm{mmHg}$, while pre-operative median $\left(\mathrm{Q}_{1}, \mathrm{Q}_{3}\right)$ number of hypotensive medications was 2 (1, 2). After operation, statistically significant lower IOPs were seen at all time points. Mean IOP at 6, 12, and 18 months were 12.4, 11.0, and $11.9 \mathrm{mmHg}$ respectively. In addition, post-operative number of hypotensive medication was also statistically significantly less than baseline, with median $\left(\mathrm{Q}_{1}, \mathrm{Q}_{3}\right) 1(0,1)$, and $20 \%$ of these eyes were medication-free.

Conclusion: Phacoemulsification and IOL implantation surgery alone in chronic ACG patients without extensive PAS can significantly lower IOP up to 18 months postoperatively.
\end{abstract}

Keywords: Angle-closure, glaucoma, intraocular pressure, phacoemulsification, peripheral anterior synechiae

Glaucoma is the leading cause of visual impairment and blindness world wide [1]. Chronic angle closure glaucoma (CACG) is the dominant glaucoma type in Asia [2]. Age, female gender, and family history are important risk factors. Anterior segment morphology, lens position and its relationship to other ocular structure are also responsible for angle closure mechanism.

Correspondence to: Visanee Tantisevi, MD, Department of Ophthalmology, Faculty of Medicine, Chulalongkorn University, Bangkok 10330, Thailand.E-mail: Tvisanee@gmail.com
Frederico et al. [3] used Ultrasound Biomicroscope to evaluate quantitative change of anterior segment in CACG patients after phacoemulsification with intraocular lens (IOL) insertion surgery (phaco/IOL). Assuming that central anterior chamber depth increased approximately 30\% after surgery, they took anterior chamber angle width to be approximately $50 \%$ of the initial value. Many studies showed that intraocular pressure (IOP) significantly decreased after phaco/IOL surgery in the primary CACG, the primary acute angle closure glaucoma (AACG), the secondary CACG, the secondary acute ACG or even in the primary angle 
closure (PAC) [4-8]. However, most reports did not specify quantitative data of peripheral anterior synechiae (PAS).

In accordance to ISGEO (International Society of Geographical and Epidemiological Ophthalmology) criteria, non-visible trabecular meshwork for 270 degrees or more is an important statement for primary angle closure suspect (PACS), primary angle closure (PAC), and primary angle closure glaucoma (PACG) [9]. However, following SEAGIG (South East Asia Glaucoma Interest Group) epidemiological guideline, an eye in which appositional contact between peripheral iris and posterior trabecular meshwork is present at 180-270 degrees of the posterior trabecular meshwork that cannot be seen gonioscopically is recognized PACS [10].

In this study, we evaluated IOP after phacoemulsification with IOL implantation in CACG patients without extensive peripheral anterior synechiae. In the evaluation, CACG subjects, with accumulative PAS equal to or less than 180 degrees were defined non-extensive.

\section{Materials and methods}

A retro-prospective study was conducted under the approval by the Ethics Committee of Faculty of Medicine, Chulalongkorn University.

Medical records of CACG patients who had undergone phacoemulsification with IOL implantation at King Chulalongkorn Memorial Hospital between May 2002 and June 2007 were reviewed. The following criteria were met. Letter of permission for collecting patient data from the hospital director was obtained. For patients who had their follow-up timepoint while the study was on-going signed an informed consent to proceed for this study.

Inclusion criteria for eligible subjects were CACG patients with clinically significant cataract, gonioscopic records showed accummulative PAS $\leq 180$ degrees with laser/surgical peripheral iridotomy $\geq$ three months prior to this operation and no other previous intraocular surgeries. We excluded secondary CACG patients who had peri-operative complications and/or further interventions related to those complications, or patients who did not pay their visits along with the study protocol.

Pre-operative data included gender, age, degrees of PAS extent, IOP by applanation tonometry (mmHg) and number of anti-glaucoma medications. Postoperative data included IOP at $1,3,6,12$, and
18 months, and number of anti-glaucoma medication at 18 months.

Primary outcome was post-operative IOP at 6 , 12, and 18 months. Repeated measures ANOVA at p-value $=0.05$ was used for these comparisons. Secondary outcome was post-op number of antiglaucoma medications and Wilcoxson Signed Rank test was utilized to compare pre and post-operative medication numbers at 18 months. Systemic drugs usage was separately categorized for comparison between pre- and post-operation at 18 months by using McNemar test.

\section{Results}

Twenty-five eyes from 25 patients were included in this study. Baseline characteristics of 25 patients are shown in Table 1.

Pre-operative mean $( \pm S D)$ IOP and median $\left(Q_{1}\right.$, $\mathrm{Q}_{3}$ ) number of anti-glaucoma medications were 15.6 $( \pm 4.1) \mathrm{mmHg}$ and $2(1,2)$. At 6,12 , and 18 months after operation, mean $( \pm S D)$ IOP reduced to 12.4 $( \pm 2.7), 11.0( \pm 2.8)$, and $11.9( \pm 3.0) \mathrm{mmHg}$, respectively, which was statistically significant $(\mathrm{p}<0.05)$. In addition, our recorded IOP (mean \pm SD) at one and three months post-operation revealed a considerable decrease in IOP to $11.7( \pm 2.4)$ and 12.8 ( \pm 3.4$) \mathrm{mmHg}$, respectively, compared with preoperative baseline. This did not seem much different from the later follow-up IOPs. Mean IOP of pre- and post-operation are shown in Fig. 1. Figure 2 shows 95\% confidence interval (CI) of pre- and post-operative IOP (mmHg).

Table 1. Baseline characteristics of patients.

\begin{tabular}{lc}
\hline \multicolumn{2}{c}{ Number of subjects = 25 } \\
\hline Age \\
$\quad$ Mean \pm SD & $73.0 \pm 8.4$ \\
$\quad$ Range & $47-86$ \\
Male/female (\%) & $2 / 23(8 / 92)$ \\
PAS $<$ 90 degrees (\%) & $17(68)$ \\
$\begin{array}{l}\text { Pre-operative IOP (mmHg) } \\
\quad \text { Mean } \pm \text { SD }\end{array}$ & $15.6 \pm 4.1$ \\
$\begin{array}{l}\text { Pre-operative number of topical } \\
\text { hypotensive medications }\end{array}$ & \\
$\quad$ Median (Q1, Q3) & $2(1,2)$ \\
\hline
\end{tabular}

$\mathrm{IOP}=$ intraocular pressure, $\mathrm{PAS}=$ peripheral anterior synechiae. 


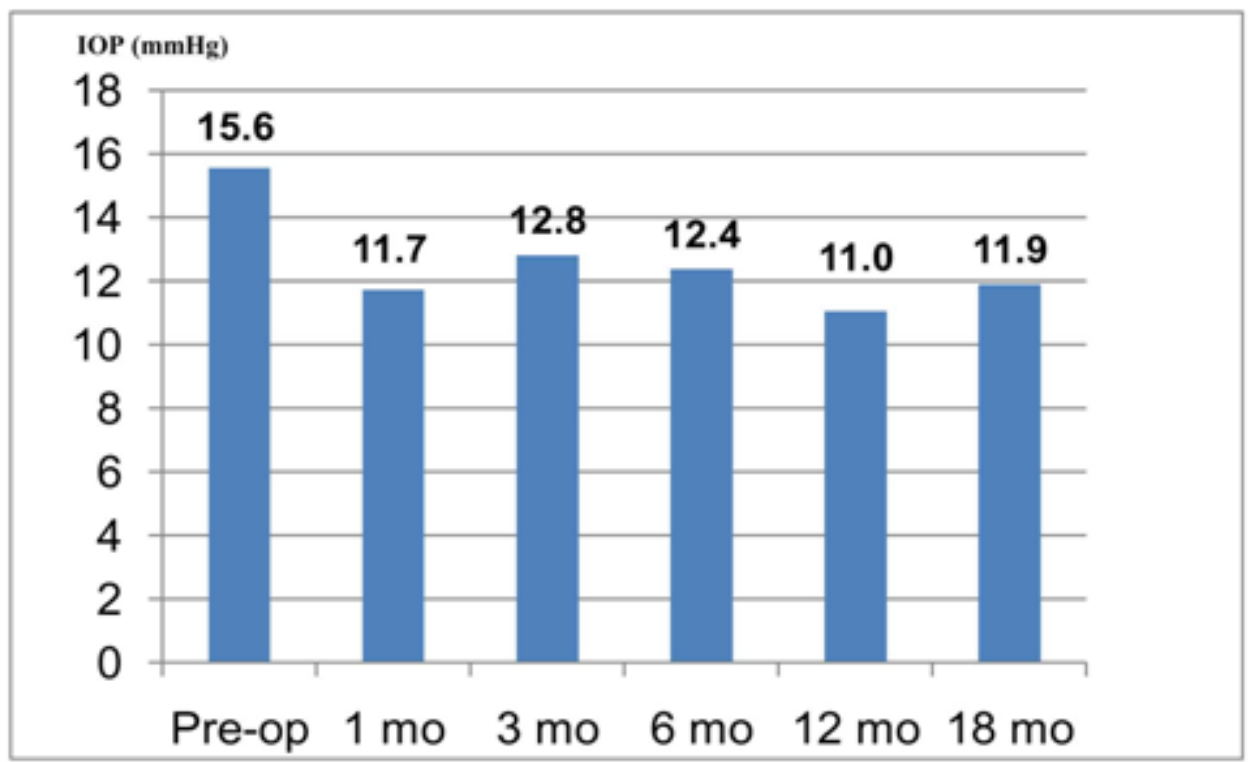

Fig. 1 Mean intraocular pressure (IOP, mmHg) of pre-and post-operation (1, 3, 6, 12, and18 months).

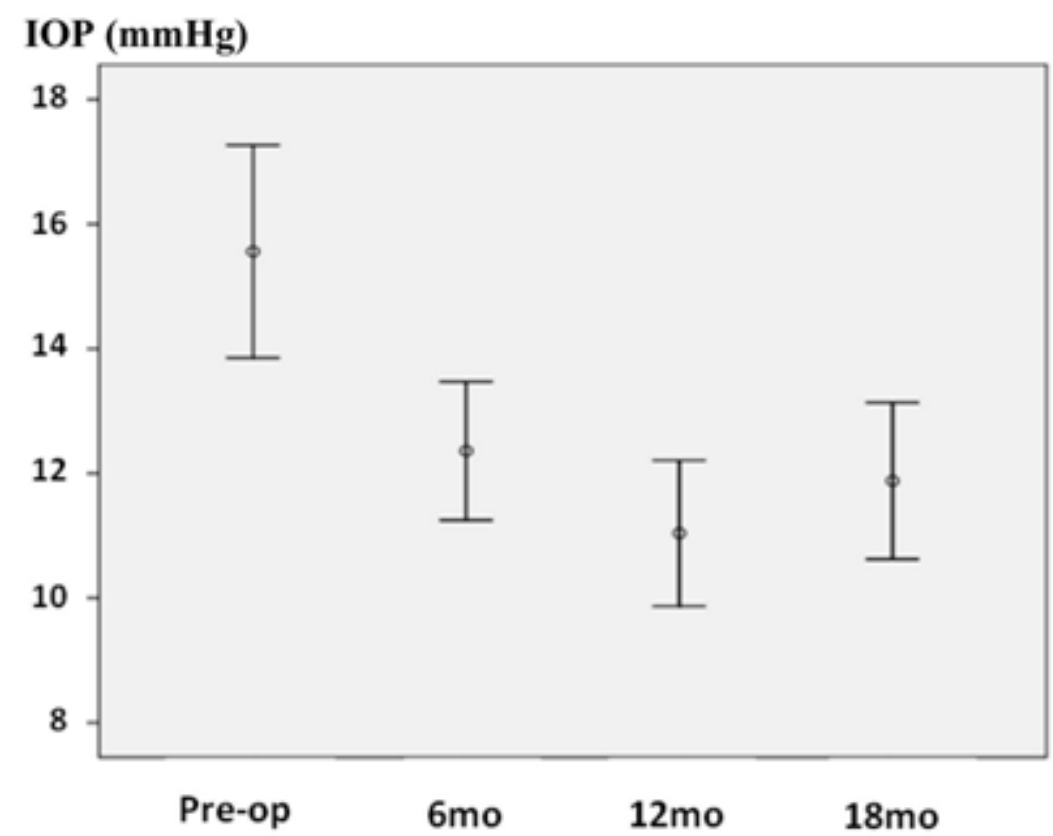

Fig. 295\% confidence interval (CI) of intraocular pressure (IOP, mmHg) of pre-and post-operation (6, 12, and 18 months).

Number of topical hypotensive medications outcome was reduced significantly $(\mathrm{p}<0.001)$ with median $\left(\mathrm{Q}_{1}, \mathrm{Q}_{3}\right) 1(0,1)$ at 18 months post-operatively (Table 2). Interestingly, at both pre- and postoperation, no systemic hypotensive medications usage was recorded in these subjects. In addition, five out of 25 (20\%) were free of glaucoma medication after their operations.

\section{Discussion}

ACG is predisposed to occur at the anterior segment of the eye [11, 12]. Lens component is an important factor of mechanisms involved in ACG. According to Pereira et al. [3], and Hayashi et al. [13], ], after cataract extraction and IOL implantation, the width of anterior chamber angle and depth of the anterior chamber increases significantly in eyes with 
Table 2. Median $\left(\mathrm{Q}_{1}, \mathrm{Q}_{3}\right)$ number of topical hypotensive medication.

\begin{tabular}{lcc}
\hline & Median $\left(\mathbf{Q}_{\mathbf{1}}, \mathbf{Q}_{\mathbf{3}}\right)$ & P-value \\
\hline Pre-operation & $2(1,2)$ & \\
$\begin{array}{l}\text { Post-operation } \\
\text { (18 months) }\end{array}$ & $1(0,1)$ & $\mathrm{p}<0.001$ \\
& & \\
\hline
\end{tabular}

similarly to eyes with open angle glaucoma and normal eyes.

In our study, the mean age of patients was 74 years old, and the percentage of women was far greater than men (female:male $=12.5: 1$ ). This tendency is consistent with previous epidemiologic studies [3, 4, 14-18] where prevalence of women was found in ACG patients. Many studies have shown that in eyes with ACG phacoemulsification and IOL implantation appeared to decrease IOP [3-7, 1719]. However, most of those results were baseline and end-point follow-up comparison. Pre-operative PAS extent was not conclusive. In our study where subjects with PAS equal to or less than 180 degrees were recruited, not only IOPs decreased significantly at six, 12 and 18 months after surgery, but also achieved considerably lower level than pre-operation one and three months after.

Euswas et al. [17] studied IOP following phacoemulsification and IOL implantation in CACG patients for six months. In all the patients, postoperative IOP was statistically reduced at any PAS extent, presence of sub-group of post-operative IOP with PAS $\leq 180$ degrees. Comparing to the our result at the same time-point of six months post-operation, our IOP level was reduced more than theirs, that is 12.4/15.6 mmHg (20.5\% reduction) and 18.1/20 $\mathrm{mmHg}$ (9.5\% reduction), respectively. Several factors might have influenced the different outcomes. Firstly, the proportion of subjects with PAS $<90$ degrees in our study was about two-third. This implies that most of our patients have considerable remaining function of trabecular meshwork outflow capacity. Secondly, our study did not collect the number of medications used at the reciprocal time-point. This could have affected measured IOPs at that time.

Two-third of our eligible subjects had accumulative PAS less than 90 degrees. It led to question if our results would be similar to those found in the primary open angle glaucoma (POAG). Mathalone et al. [20] studied the effect of clear cornea sutureles phacoemulsification with IOL in wellcontrolled POAG and control patients, in which both groups were followed at 12 and 24 months. They reported that phacoemulsification could reduce IOP from baseline in both groups at both follow-up times. In POAG subjects, IOP level was $9.2 \%$ and $11.7 \%$ lower than baseline at 12 and 24 months, and medications were reduced from mean baseline 1.1 to 0.65 at 12 months and 1.5 to 1.1 at 24 months. Interestingly, $47 \%$ and $38 \%$ in 12 months and 24 months groups, respectively, did not require postoperative hypotensive medications. In the report by Shingelton et al. [21], a small but significant decrease in IOP after clear corneal phacoemulsification with IOL implantation in glaucoma $(1.4 \pm 3.3 \mathrm{mmHg}$ ) group, no-glaucoma $(1.7 \pm 3.1 \mathrm{mmHg})$ group and glaucoma suspect (1.4 $\pm 4.2 \mathrm{mmHg}$ ) group were revealed without change in medication number at three years after operations. The same features were reported in other studies [22-24]. It seems that phacoemulsification in open-angle glaucoma showed less IOP reduction effect compared with those in angle closure in our study. Nonetheless, number of medication in our and their studies could not be disclosed because the statistics used were not the same.

In conclusion, phacoemulsification and IOL implantation alone in CACG patients without extensive PAS can significantly lower IOP and medication use up to 18 months post-operation. However, medications seemed partially necessary to maintain satisfactory IOP level.

The authors have no conflict of interest to report.

\section{References}

1. Quigley HA. Number of people with glaucoma worldwide. Br J Ophthalmol. 1996; 80:389-93.

2. Simmons ST, Cioffi GA, Gross RL, Myers JS, Netland PA, Samples JR, et al. Angle-closure glaucoma. In: Leisegang TJ, Skuta GL, Cantor LB, editors. Basic and clinical science course. San Francisco:Am Acad Ophthalmol. 2005-2006. p.119-46.

3. Pereira FA, Cronemberger S. Ultrasound biomicroscopic study of anterior segment changes after phacoemulsification and foldable intraocular lens implantation. Ophthalmology. 2003;110:1799-806.

4. Hayashi K, Hayashi F, Nakao F, Hayashi F. Effect of cataract surgery on intraocular pressure control in glaucoma patients. J Cataract Refraction Surgery. 2001; 27:1779-86. 
5. Ge J, Guo Y, Lui Y, Lin M, Zhou Y, Chen B, et al. New management of angle-closure glaucoma by phacoemulsification with foldable posterior chamber intraocular lens implantation (in Chinese). Yan Ke Xue Bao. 2000; 16:22-8.

6. Ge J, Guo Y, Lui Y. Preliminary clinical study on the management of angle-closure glaucoma by phacoemulsification with foldable posterior chamber intraocular lens implantation (in Chinese). Zhonghua Yan Ke Za Zhi. 2001; 37: 355-8.

7. Hata H, Yamane S, Hata S, Shiota H. Preliminary outcomes of primary phacoemulsification plus intraocular lens implantation for primary angle-closure glaucoma. J Med Invest. 2008; 55:287-91.

8. Zhang X, Teng L, Li A, Du S, Zhu Y, Ge J. The clinical outcomes of three surgical managements on primary angle-closure glaucoma (in Chinese). Yan Ke Xue Bao. 2007; 23:65-74.

9. Paul J Foster, Burhmann R, Quigley HA, Johnson GJ. The definition and classification of glaucoma in prevalence surveys. Br J Ophthalmol. 2002; 86: 238-42.

10. Definition of terms. In: Sasidharan R, editor. Asia Pacific Glaucoma Guidelines. South East Asian Glaucoma Interest Group. $2^{\text {nd }}$ ed. Hong Kong:Scientific Communications International; 2008. p.104

11. Lowe RF. Aetiology of the anatomical basis for primary angle-closure glaucoma. Br J Ophthalmol. 1970; 54:161-9.

12. Alsbirk $\mathrm{PH}$. Anterior chamber depth and primary angle closure glaucoma. An epidemiologic study in Greenland Eskimos. Acta Ophthalmol Copenh. 1975; 53:89-104.

13. Hayashi K, Hayashi H, Nakao F, Hayashi F. Changes in anterior chamber angle width and depth after intraocular lens implantation in eyes with glaucoma. Ophthalmology. 2000; 107:698-703.

14. Lai JS, Tham CC, Chan JC. The clinical outcomes of cataract extraction by phacoemulsification in eyes with primary angle-closure glaucoma and co-existing cataract: a prospective case series. J Glaucoma. 2006; 15:47-52.
15. Alsbirk PH. Primary angle-closure glaucoma: oculometry, epidemiology, and genetics in a high risk population. Acta Ophthalmol Suppl. 1976; 127:89-104.

16. Seah SKL, Foster PJ, Chew PTK, Jap A, Oen F, Fam HB, et al. Incidence of acute primary angle-closure glaucoma in Singapore: an island-wide survey. Arch Ophthalmol. 1997; 115:1436-144.

17. Euswas A, Warrasak S. Intraocular pressure control following phacoemulsification in patients with chronic angle closure glaucoma. J Med Assoc Thai. 2005; 8(Suppl 9):121-5.

18. Pachimkul P, Intajak Y. Effect of lens extraction on primary angle closure in a Thai population. J Med Assoc Thai. 2008; 91:303-8.

19. Wu JH, Liu L, Zhou SH, Zhong M, Peng YJ, Shen W, et al. Management of primary angle-closure glaucoma by phacoemulsification with foldable posterior chamber intraocular lens implantation (in Chinese). Zhejiang Da Xue Xue Bao Yi Xue Ban. 2008; 37:407-12.

20. Mathalone N, Hyams M, Neiman S. Long-term intraocular pressure control after clear corneal phacoemulsification in glaucoma patients. J Cataract Refract Surg. 2005; 31:479-83.

21. Shingleton BJ, Pasternack JJ, Hung JW, O’Donoghue MW. Three and five year changes in intraocular pressures after clear corneal phacoemulsification in open angle glaucoma patients, glaucoma suspects, and normal patients. J Glaucoma. 2006; 15:494-8.

22. Kim DD, Doyle JW, Smith MF. Intraocular pressure reduction following phacoemulsification cataract extraction with posterior chamber lens implantation in glaucoma patients. Ophthalmic Surg Lasers. 1999; 30:37-40.

23. Hudovernik M, Pahor D. Intraocular pressure after phacoemulsification with posterior chamber lens implantation in open-angle glaucoma. Klin Monatsbl Augenheilkd. 2003; 220:835-9.

24. Pohjalainen T, Vesti E, Uusitalo RJ, Laatikainen L. Phacoemulsification and intraocular lens implantation in eyes with open-angle glaucoma. Acta Ophthalmol Scand. 2001; 79:313-6. 